\title{
CD300b regulates the phagocytosis of apoptotic cells via phosphatidylserine recognition
}

\author{
Y Murakami ${ }^{1}$, L Tian ${ }^{1}$, OH Voss ${ }^{1}$, DH Margulies ${ }^{2}$, K Krzewski $^{1}$ and JE Coligan ${ }^{*, 1}$
}

The CD300 receptor family members are a group of molecules that modulate a variety of immune cell processes. We show that mouse CD300b (CLM7/LMIR5), expressed on myeloid cells, recognizes outer membrane-exposed phosphatidylserine (PS) and does not, as previously reported, directly recognize TIM1 or TIM4. CD300b accumulates in phagocytic cups along with F-actin at apoptotic cell contacts, thereby facilitating their engulfment. The CD300b-mediated activation signal is conveyed through CD300b association with the adaptor molecule DAP12, and requires a functional DAP12 ITAM motif. Binding of apoptotic cells promotes the activation of the PI3K-Akt kinase pathway in macrophages, while silencing of CD300b expression diminishes PI3KAkt kinase activation and impairs efferocytosis. Collectively, our data show that CD300b recognizes PS as a ligand, and regulates the phagocytosis of apoptotic cells via the DAP12 signaling pathway.

Cell Death and Differentiation (2014) 21, 1746-1757; doi:10.1038/cdd.2014.86; published online 18 July 2014

In both developing and mature multicellular organisms, large numbers of apoptotic cells are continually generated and must be cleared by neighboring cells or 'professional' phagocytes. $^{1-4}$ If not properly cleared, they become necrotic, pro-inflammatory and immunogenic, potentially leading to the development of autoimmune diseases, such as systemic lupus erythematous (SLE).$^{5-8}$ Therefore, phagocytes possess sensing systems to facilitate the clearance of apoptotic cells. ${ }^{1-3}$ Once guided to their location by diffusible 'find me' signals, phagocytes recognize apoptotic cells through their display of characteristic cell surface molecules ('eat me' signals). ${ }^{4,7}$ The most common signal promoting phagocytosis is the recognition of phosphatidylserine (PS), which when exposed on the outer leaflet of the plasma membrane signals phagocytes to engulf apoptotic cells. ${ }^{2}$ Multiple receptors for PS exist on phagocytic cells, although not necessarily simultaneously; these include stabilins, ${ }^{9,10} \mathrm{~T}$ cell Ig mucin (TIM) 1 and TIM4, ${ }^{11,12}$ BAI1, ${ }^{13}$ MFGE8, which bridges PS to integrin $\alpha v \beta 3,{ }^{14}$ and Protein $S$ and Gas6, which bridge PS to TAM receptors. ${ }^{15}$ Recently, we and others demonstrated that the CD300 family members, human and mouse CD300a, ${ }^{16,17}$ and mouse CD300f, ${ }^{18,19}$ also bind PS, and their expression regulates apoptotic cell phagocytosis.

The CD300 family contains both activating and inhibitory receptor members. ${ }^{20} \mathrm{CD} 300 \mathrm{~b}$ has a short intracellular tail and gains activation potential by association with DNAX activating protein of $12 \mathrm{kDa}$ (DAP12) or DAP10 adaptor molecules. ${ }^{21}$ $\mathrm{CD} 300 \mathrm{~b}$ is predominantly expressed on myeloid cells, including neutrophils, macrophages and mast cells. Antibody cross-linking of human and mouse CD300b has been shown to induce the release of inflammatory cytokines from mast cells. ${ }^{21}$ The ligand for CD300b remains a matter of debate. A recent study found that a soluble form of CD300b, released in response to Toll-like receptor ligation, recognizes unknown ligands on the surface of macrophages, resulting in the release of inflammatory cytokines. ${ }^{22}$ Others have identified the PS-binding receptors TIM1 and TIM4 as endogenous ligands for CD300b, but not PS itself. ${ }^{23}$

Here, we show that CD300b binds to PS, and recognizes PS on TIM1 or TIM4 expressing cells rather than TIM1 or TIM4 alone. We found that CD300b promotes PS-dependent apoptotic cell phagocytosis upon ectopic expression in cell lines, without the need for additional PS receptors. In addition, CD300b-mediated phagocytosis requires the association of the adaptor protein DAP12 for effective signaling. Inhibition of CD300b function by either anti-CD300b antibody treatment or siRNA transfection significantly decreases macrophagedependent phagocytosis of apoptotic cells. Furthermore, CD300b silencing in macrophages severely impairs the apoptotic cell-induced phosphorylation of PI3K, Akt and Syk, but not Erk. Thus, our data show that CD300b is an activating receptor that has an important role in macrophage-mediated clearance of apoptotic cells.

\section{Results}

CD300b binds phosphatidylserine in the absence of TIM1 or TIM4. We analyzed the binding of recombinant murine CD300b to phospholipids known to associate with CD300 molecules ${ }^{16-19,24-26}$ by surface plasmon resonance

\footnotetext{
${ }^{1}$ Receptor Cell Biology Section, Laboratory of Immunogenetics, National Institute of Allergy and Infectious Diseases, National Institutes of Health, Rockville, MD, USA and ${ }^{2}$ Molecular Biology Section, Laboratory of Immunology, National Institute of Allergy and Infectious Diseases, Bethesda, MD, USA

${ }^{*}$ Corresponding author: JE Coligan, 12441 Parklawn Drive, Twinbrook II, Room 205, Rockville, MD 20852, USA. Tel: +301 4968247 ; Fax: +301 480 2818; E-mail: jcoligan@niaid.nih.gov

Abbreviations: DAP12, DNAX activating protein of $12 \mathrm{kDa}$; TIM, T cell Ig mucin; POPC, 1-palmitoyl-2-oleoyl-sn-glycero-3-phosphocholine; POPE, 1-palmitoyl-2oleoyl-sn-glycero-3-phosphoethanolamine; POPS, 1-palmitoyl-2-oleoyl-sn-glycero-3-phospho-L-serine, 1,2-dioleoyl-sn-glycero-3-phosphocholine; DOPC, 1,2-dioleoylsn-glycero-3-phosphoserine; DOPS, 1,2-dioleoyl-sn-glycero-3-phosphoethanolamine; DHPE, N-(biotinoyl)-1,2-dihexadecanoyl-sn-glycero-3-phosphoethanolamine, triethylammonium salt, biotin-labeled; SPR, surface plasmon resonance; PS, phosphatidylserine; PC, phosphatidylcholine; PE, phosphatidylethanolamine; ITAM, immunoreceptor tyrosine-based activation motif

Received 24.2.14; revised 14.5.14; accepted 21.5.14; Edited by M Piacentini; published online 18.7.14
} 
(SPR), and found that CD300b-Fc bound to phosphatidylserine (PS)-containing liposomes, but not those containing phosphatidylcholine (PC), phosphatidylethanolamine (PE) or ceramide (Figure 1a; Supplementary Figure 1). A control protein, NITR-Fc, ${ }^{24}$ failed to bind to any liposomes (Figure 1b). CD300b-Fc bound to PS-containing lipsomes as indicated by the steady rise in Resonance Units (RU) during analyte injection. This interaction was stable through the washout phase. No significant binding to PC- or PE-coated liposomes was observed, although some deterioration of these biosensor surfaces during the washout phase was noted.

PS is exposed on the cell surface during apoptosis. To examine whether CD300b binds to apoptotic cells, dexamethasone-induced apoptotic thymocytes were simultaneously stained with CD300b-Fc and Annexin V. CD300b-Fc specifically recognized Annexin $\mathrm{V}^{+}$apoptotic thymocytes but not Annexin $\mathrm{V}^{-}$cells (Figure 2a). To confirm whether CD300b binding to dead cells is dependent on PS, apoptotic cells were pretreated with recombinant unlabeled Annexin $\mathrm{V}$ to mask the exposed PS, and then CD300b-Fc binding was analyzed. Annexin V pretreatment significantly decreased the binding of CD300b-Fc in a dose-dependent manner (Figure 2b). Moreover, the binding of CD300b-Fc to apoptotic cells was inhibited by preincubation with PS- but not by PE- or PC-coated liposomes (Figure 2c), further supporting the notion that CD300b binding to apoptotic cells requires the recognition of $P S$. Because the binding of Annexin $V$ and other proteins to PS involves the presence of $\mathrm{Ca}^{2+}$, we investigated whether CD300b binding to PS also required a metal ion. Treatment with EDTA or EGTA reduced the binding of $\mathrm{CD} 300 \mathrm{~b}-\mathrm{Fc}$ to apoptotic cells, indicating the requirement for $\mathrm{Ca}^{2+}$ (Figure 2d). These findings demonstrate that PS is a direct and specific ligand for CD300b.

CD300b binding to TIM1 and TIM4 depends on phosphatidylserine. A previous report demonstrated that CD300b recognized TIM1- or TIM4-expressing Ba/F3 cells. ${ }^{23}$ To investigate further the binding of CD300b to TIM1 and TIM4, we generated TIM1- or TIM4-expressing Ba/F3 cell lines. We confirmed that Annexin V, as well as CD300b-Fc, bound to both cells but not EV-transduced cells (Figures $3 a$ and b; Supplementary Figures $2 a$ and b), and that EDTA treatment abolished that binding (Supplementary Figure $2 \mathrm{c}$ ). Interestingly, the CD300b-Fc binding or Annexin V staining was significantly reduced when cells were pretreated with recombinant unlabeled Annexin V (Figure 3c; Supplementary Figure 2d). These findings suggest that the binding of CD300b to TIM1- or TIM4-expressing cells was PS-dependent. TIM1- or TIM4-expressing Ba/F3 cells are known to capture PS-expressing exosomes; ${ }^{12}$ thus it is plausible that CD300b does not bind TIM1 or TIM4 directly as previously proposed, ${ }^{23}$ but rather associates with these proteins indirectly through associated PS-containing exosomes, or other PS-containing membrane fragments. With this line of reasoning, we hypothesized that the binding affinity of CD300b to TIM1- or TIM4-expressing Ba/F3 cells would increase after the addition of PS-coated liposomes. To test a
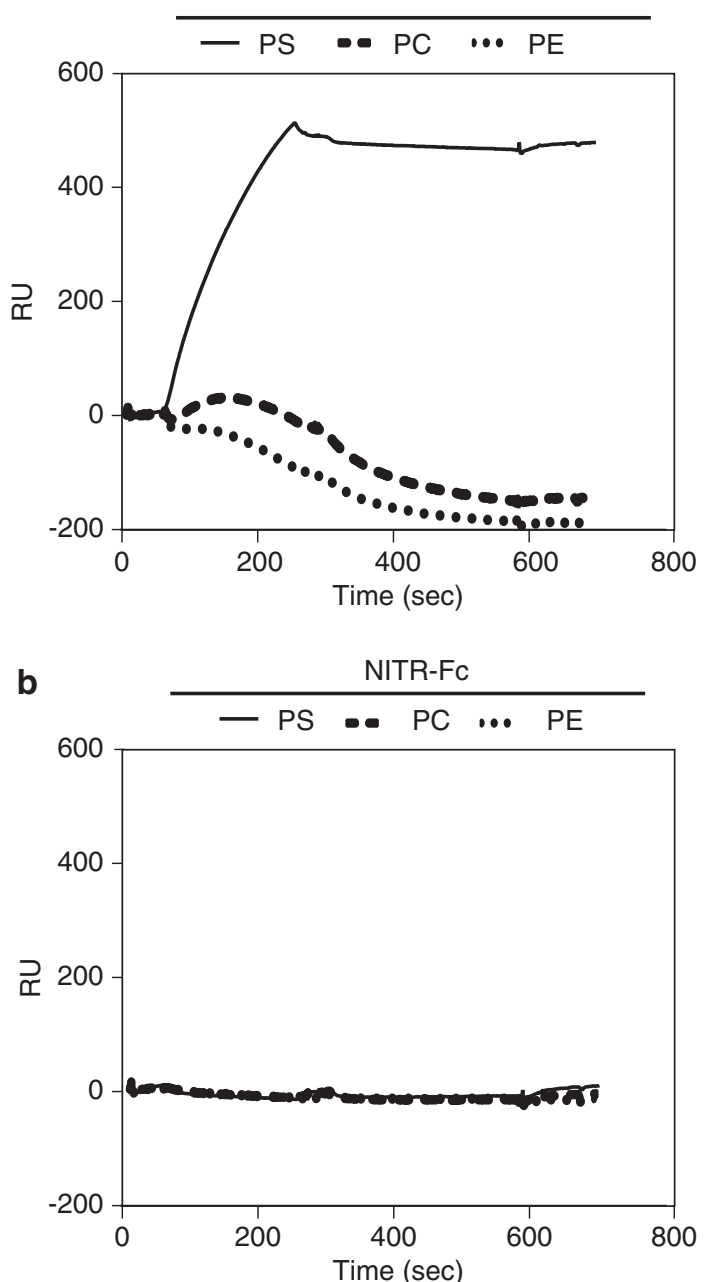

Figure $1 \mathrm{CD} 300 \mathrm{~b}$ binds phosphatidylserine-containing liposomes. (a and $\mathbf{b}) \mathrm{PS}$ liposomes (DOPC/POPS at 4:1 ratio), PC liposomes (DOPC/POPC at 4:1 ratio) or $P E$ liposomes (DOPC/POPE at 4:1 ratio) were immobilized on a $L 1$ biosensor. Liposome binding was monitored in resonance units (RU) using $10 \mu \mathrm{g}$ of CD300bFc (a) or NITR-Fc chimera proteins (b). Binding was initiated at $60 \mathrm{~s}$ and the washout (dissociation phase) begun at $240 \mathrm{~s}$. Data are a representative of three experiments

this possibility, biotin-labeled liposomes were incubated with TIM1- or TIM4-expressing Ba/F3 cells. TIM1- or TIM4-expressing cells bound 10-fold more PS liposomes than EV-transduced control cells (Figure 3d; Supplementary Figure 2e). Importantly, and consistent with our hypothesis, addition of PS-coated liposomes caused an increase in CD300b-Fc binding to TIM1- or TIM4-expressing cells, when compared with EV-transduced cells or cells treated with PCcoated liposomes (Figure 3e; Supplementary Figure 2f). Thus, the observed binding of CD300b-Fc to TIM1 or TIM4 is likely not direct, but occurs via bound PS-containing cell fragments and/or exosomes. To address directly whether CD300b recognizes TIM1, the interaction between TIM1 and CD300b-Fc was analyzed by SPR in the presence or absence of liposomes. Although the association level of CD300b-Fc to TIM1 was similar to reactions containing the 


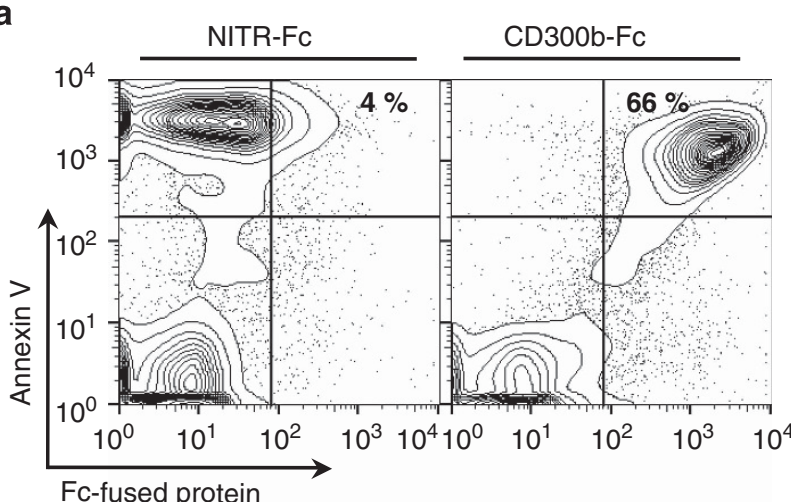

b
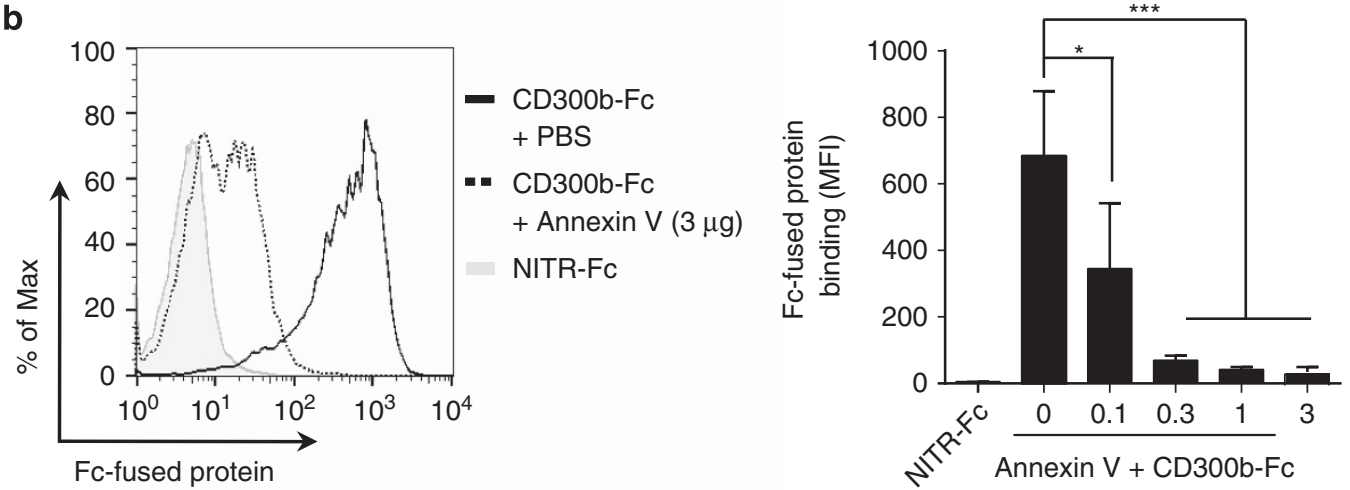

c
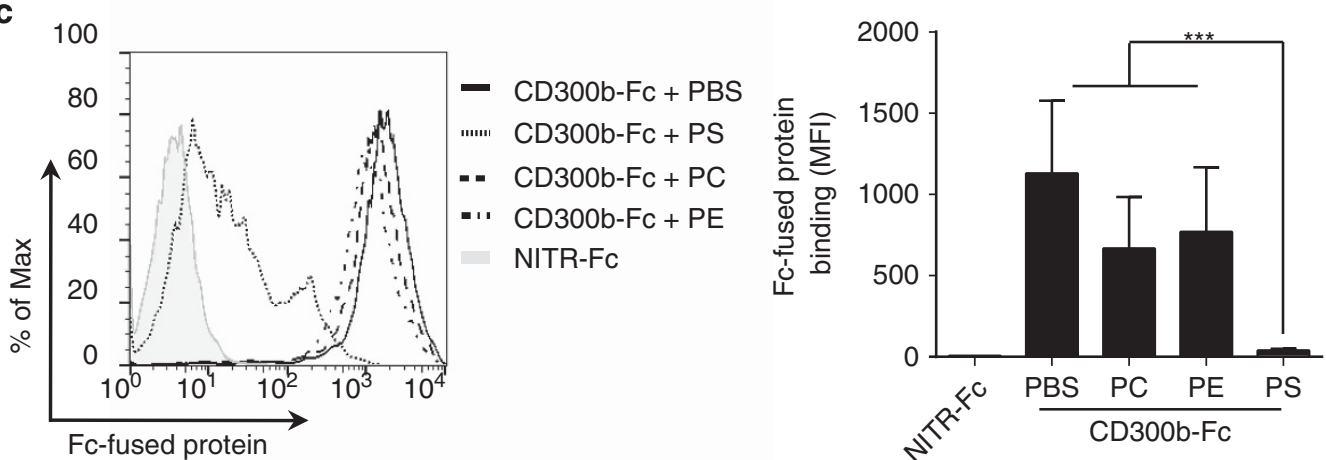

c

- CD300b-Fc + PBS

"nu! $\quad \mathrm{CD} 300 \mathrm{~b}-\mathrm{Fc}+\mathrm{PS}$

- CD300b-Fc+PC

$\because$ CD300b-Fc + PE NITR-FC

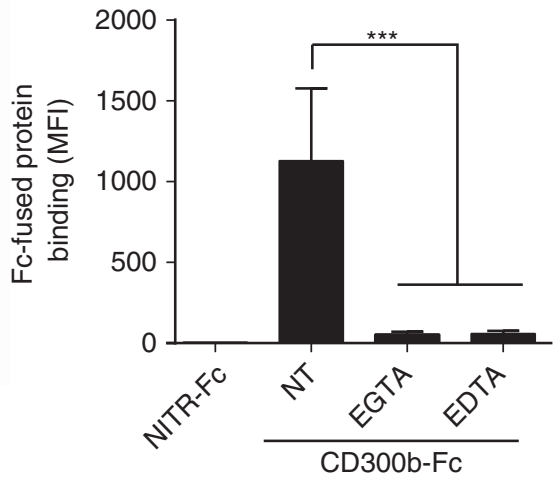


NITR-Fc control protein (Figure 3f), CD300b-Fc binding to TIM1 only occurred in the presence of PS- but not PC-coated liposomes (Figure $3 \mathrm{~g}$ ). Taken together, our data suggest that CD300b does not recognize TIM1 or TIM4 directly, but can interact with these proteins indirectly through bound PSexpressing cell fragments.

CD300b regulates the phagocytosis of apoptotic cells through engagement of DAP12 and activation of the PI3K signaling pathway. To investigate whether CD300b mediates phagocytosis, CD300b- and/or DAP12-encoding lentiviruses were used to transduce L929 fibroblasts. L929 cells are endogenously deficient in the expression of the majority of PS receptors, like CD300b, CD300f, TIM1, TIM4, BAl1 or Stabilin2, which are only detectable upon transfection (Supplementary Figures 3a and b). However, L929 cells express low levels of Axl and MerTK, which likely are responsible for the observed baseline of apoptotic cell phagocytosis (Figures 4 and 5; Supplementary Figure 3b; and Tian et al. ${ }^{19}$ ). Microscopic (Figure 4a) and flow cytometric (Figure 4b) analyses demonstrated that CD300b-DAP12expressing L929 cells efficiently engulfed PS-coated liposome beads, whereas CD300b-transduced cells showed association but not engulfment of PS-containing beads (Figures $4 \mathrm{a}$ and b). In addition, PC-coated liposome beads showed some increase in binding to CD300b and DAP12 co-expressing L929 cells, as compared with EV-, or CD300bor DAP12-transduced cells (Figure 4a), however these beads were not engulfed (Figure $4 \mathrm{~b}$ ). These findings suggest that CD300b has a low affinity towards PC-coated beads and further demonstrate that PS recognition by CD300b mediates efficient phagocytic uptake that requires the association with an adaptor molecule, like DAP12.

To determine whether CD300b promotes phagocytosis of apoptotic cells, we used apoptotic thymocytes labeled with pHrodo, a pH-sensitive dye that becomes fluorescent upon phagocytosis. ${ }^{27}$ Our findings showed that CD300b-expressing L929 cells had similar levels of phagocytosis as compared with EV- and DAP12-transduced cells. In contrast, cells co-expressing CD300b and DAP12 demonstrated a significant enhancement in the percentage of phagocytosis (Figures 5a and b). Unlike DAP12, overexpression of DAP10 with CD300b did not result in a significant increase in the level of phagocytosis (Supplementary Figure 4). Moreover, F-actin and CD300b accumulated at the contact site with the apoptotic cells, supporting the notion that CD300b directly mediates the uptake of apoptotic cells (Figure 5c). We confirmed that the CD300b-mediated phagocytosis of apoptotic cells occurred via PS recognition by showing that engulfment of apoptotic cells was inhibited in the presence of PS-coated liposomes (Figure 5d), and other PS-binding proteins, like recombinant Annexin V, CD300f-Fc or CD300b-Fc itself (Figure 5e). Furthermore, the absence or low expression of the majority of other PS receptors on L929 cells suggest that CD300b alone is sufficient to efficiently recognize PS and induce the signaling events necessary to regulate the phagocytosis of apoptotic cells, without the need to co-engage other PS receptors.

DAP12 mediates signaling via its ITAM motif. ${ }^{28}$ To confirm the importance of this motif for CD300b-mediated phagocytosis, we generated a DAP12 mutant (DAP12m), in which the ITAM tyrosine residue was mutated to a phenylalanine, abolishing the phospho-tyrosine-mediated signaling cascade. CD300b and DAP12m co-expressing L929 cells showed similar levels of phagocytosis as compared with cells expressing EV-, DAP12, DAP12m or CD300b. However, CD300b-DAP12-co-expressing cells demonstrated a significant enhancement in the level of phagocytosis, suggesting that the interaction between CD300b and DAP12, and DAP12-mediated signaling are necessary for efficient CD300b-mediated phagocytosis (Figure 5f).

To verify that CD300b has a role in phagocytosis by primary macrophages, we first pretreated peritoneal macrophages with an anti-CD300b antibody, and then the phagocytosis of apoptotic cells was analyzed by flow cytometry (Supplementary Figure 5). Our findings showed a significant reduction in the percentage of macrophages that engulfed apoptotic cells in the presence of anti-CD300b antibody treated cells as compared with cells cocultured with anti-IgGcontrol antibodies (Supplementary Figure 5a). Moreover, not only did fewer macrophages engulf apoptotic cells in the presence of anti-CD300b, but those that did stained less intensely for the presence of $\mathrm{pHrodo}^{+}$-labeled apoptotic cells (Supplementary Figures $5 b$ and $c$ ). Next, we silenced the expression of CD300b by shRNA in J774.1 macrophage cells (Figure 6a). Reduced expression of CD300b markedly impaired phagocytosis of apoptotic cells, suggesting that CD300b has an important role in the efferocytosis mediated by macrophages (Figure 6b). As previously shown (Figure 5f), co-expression of adaptor molecule, DAP12, is critical for CD300b-mediated phagocytosis. To determine which signaling pathways were activated upon CD300b-DAP12 engagement of apoptotic cells, we analyzed the phosphorylation status of several signaling molecules in $\mathbf{J 7 7 4 . 1}$ cells cultured with apoptotic thymocytes. Upon exposure to apoptotic cells, phosphorylation of Akt, Syk, Erk and PI3K was induced in

\footnotetext{
Figure $2 \mathrm{CD} 300 \mathrm{~b}$ recognition of apoptotic thymocytes is dependent on phosphatidylserine. Murine thymocytes were cultured with $10 \mu \mathrm{M}$ dexamethazone for $6 \mathrm{~h}$ to induce apoptosis. (a) Cells were incubated with $0.1 \mu \mathrm{g}$ CD300b-Fc or NITR-Fc fusion proteins and stained with Annexin V-APC and anti-human IgG Fc $\gamma$-FITC antibody. Binding of Fcchimera proteins to apoptotic thymocytes was analyzed by flow cytometry. (b) Apoptotic thymocytes $\left(0.25 \times 10^{6}\right.$ cells), pretreated with various concentrations of unlabeled Annexin V, were incubated with $0.1 \mu \mathrm{g}$ CD300b- or NITR-Fc proteins and stained with anti-human IgG Fc $\gamma$-FITC antibody. (c) PC-, PE- or PS-coated liposomes (10 $\mu$ M) were preincubated with $0.1 \mu \mathrm{g}$ CD300b- or NITR-Fc proteins and then mixed with apoptotic thymocytes. Binding of Fc-chimera proteins to the cell surface was detected using an anti-human IgG Fc $\gamma$-FITC antibody. (d) Apoptotic thymocytes were incubated with $0.1 \mu \mathrm{g}$ CD300b-or NITR-Fc proteins in the presence or absence of $5 \mathrm{mM}$ EDTA or EGTA and stained with anti-human IgG Fcy-FITC antibody. (b-d) The histograms on the left show examples of binding of Fc-fusion proteins to the apoptotic cells; the graphs on the right shows the mean fluorescence intensity (MFI) values of the bound $\mathrm{Fc}$-fused proteins, from three independent experiments and represent mean $\pm S$.E.M.; ${ }^{*} P<0.05$, ${ }^{\star \star \star} P<0.001$ (Student's $t$-test)
} 
shControl and shCD300b macrophages. However, although Erk phosphorylation levels were similar in both cell types, the phosphorylation levels of PI3K, Akt and Syk were significantly reduced in CD300b knockdown cells (Figure 6c), suggesting that CD300b-mediated signals events involve the DAP12Syk-PI3K-Akt signaling pathway. a

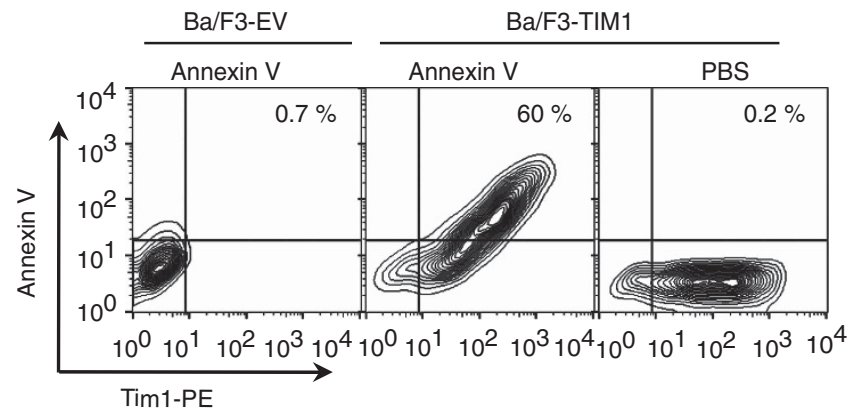

C

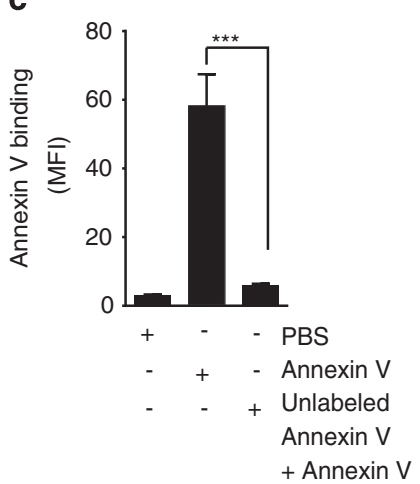

e

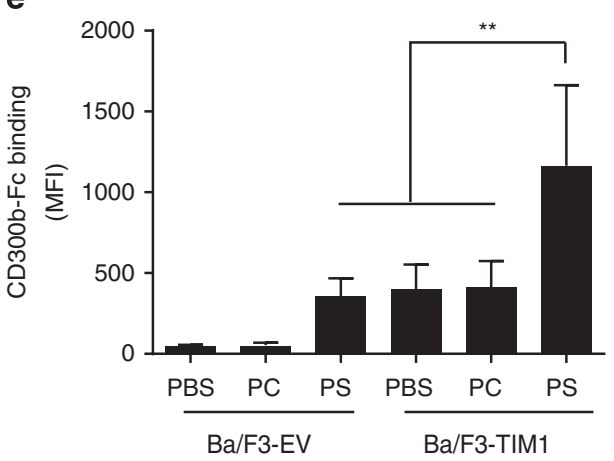

g



b

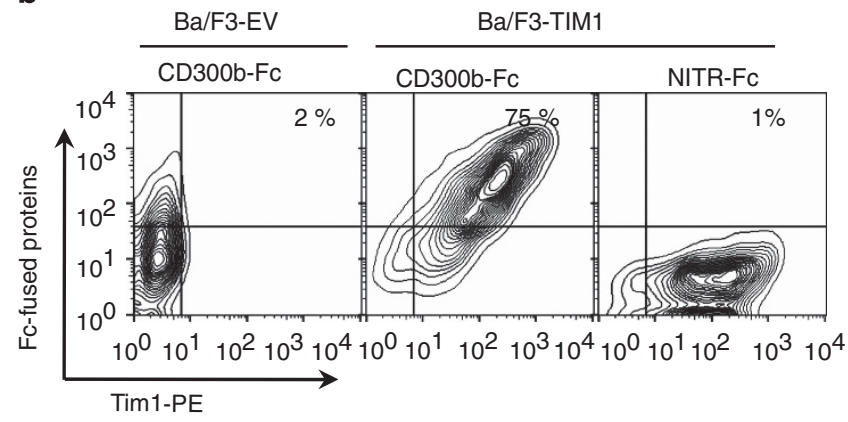

d

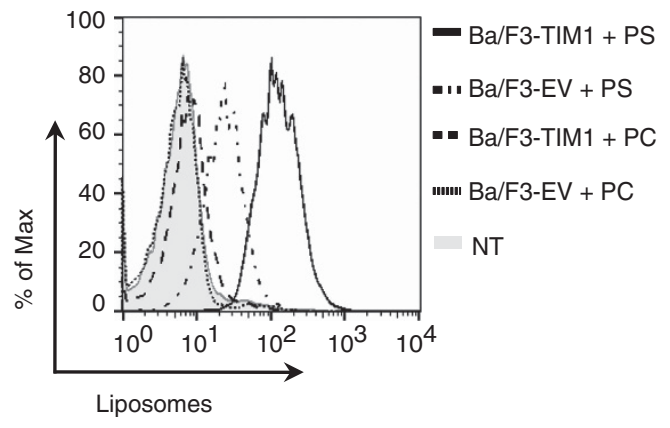

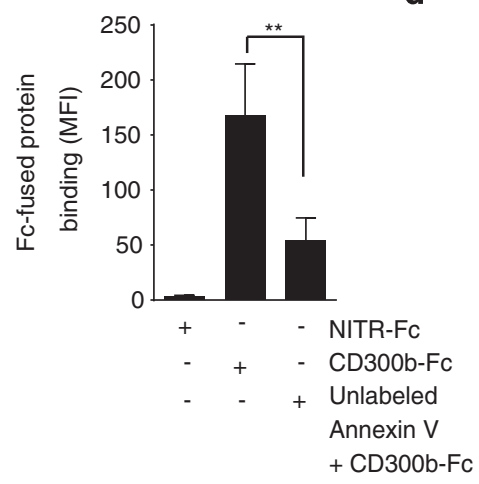

f
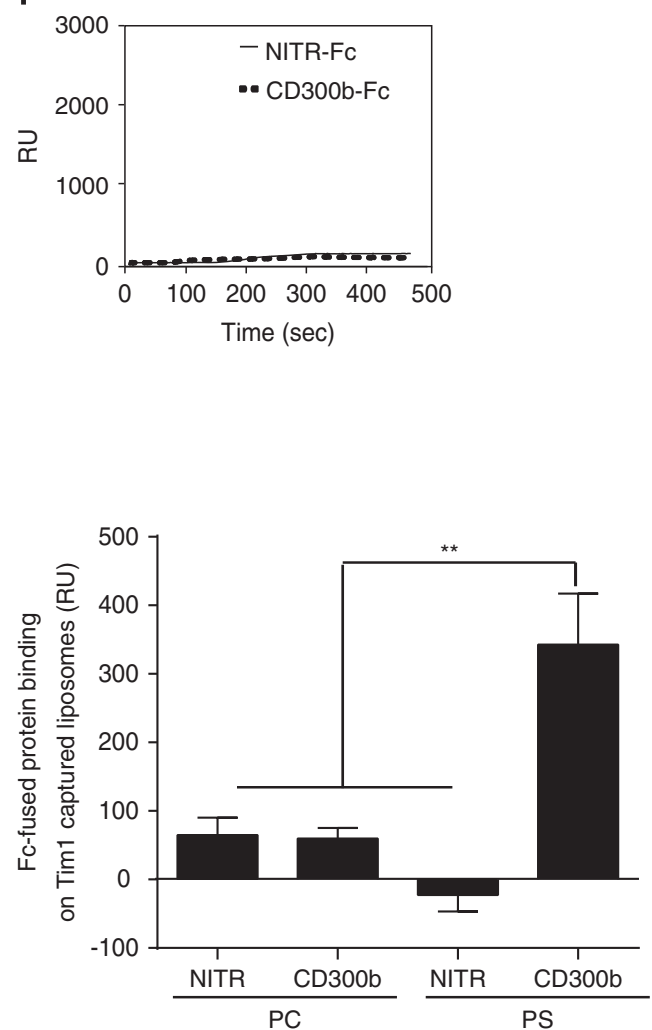


\section{Discussion}

In this study, we demonstrated that CD300b recognizes PS-coated liposomes, but not other phospholipid-containing liposomes. Moreover, we showed that CD300b binding to apoptotic cells is dependent on PS, as recombinant unlabeled Annexin V or PS-coated liposomes, but not other phospholipid-containing liposomes, blocked the CD300b-mediated engulfment of apoptotic cells.

Previously, Yamanishi et al. ${ }^{23}$ demonstrated that the PS receptors, TIM1 and TIM4, are ligands of CD300b, as TIM1- or TIM4-transduced cells were able to bind to the CD300b-Fc chimera protein. Their findings further argued against phospholipids as ligands for CD300b, due to lack of CD300b binding to any phospholipid by membrane blotting. In contrast, we demonstrate here that CD300b not only binds PS, but PS is essential for the interaction between CD300b and TIM1 or TIM4. The discrepancy in the results is most likely due to the differences in the experimental conditions. In this study, we characterize PS as a ligand of CD300b by utilizing phospholipid-coated liposomes, an approach successfully used in identifying ligands for other CD300 family members. ${ }^{18,19}$ The previous studies failed to detect PS binding when using flat surfaces, which could restrict ligand access, an experimental limitation previously shown for plates coated with phospholipids and membrane spotted phospholipids. ${ }^{24,25}$ Thus, the ligand binding by CD300 family members most likely depends on the presence or mimicking of the physiological cell surface environment.

Miyanishi et al. ${ }^{12}$ demonstrated by electron microscopy that TIM1 or TIM4 can capture exosomes with PS exposed on their outer surface. This implies that CD300b could bind to TIM1 or TIM4 directly, when in fact the association would be indirect through TIM1- or TIM4-bound PS-containing exosomes or cell fragments of apoptotic cells. This hypothesis is further supported by the fact that recombinant unlabeled Annexin $\mathrm{V}$ can efficiently block either CD300b-Fc or Annexin $\mathrm{V}$ binding to TIM1- or TIM4-expressing cells, and that the addition of PScoated liposomes enhanced the binding of CD300b to TIM1expressing cells. Purified TIM1- or TIM4-His tagged proteins do not interact with CD300b-Fc when analyzed by SPR, whereas CD300b binding to both TIM1 and TIM4 was only observed in the presence of PS-coated liposomes. Thus, we conclude that CD300b does not directly recognize TIM1 or TIM4, but recognizes PS-containing cell fragments or exosomes captured by TIM1 or TIM4.
The immune system is tightly regulated by a balance between activating and inhibitory signals. ${ }^{29-32}$ Many of these balancing signals originate from activating and inhibitory receptors of closely related family members that have identical or very similar ligand specificities, for example, NKG2A and NKG2C, ${ }^{29}$ killer-cell immunoglobulin-like receptors, ${ }^{31}$ CD28 and CTLA4, PIR-A and PIR-B. ${ }^{33}$ The CD300 receptors are another such family expressed mainly on myeloid cells. Furthermore, work from us and others showed that at least three members of this family, CD300a, ${ }^{16,26}$ CD300f, $^{18,19}$ and now CD300b, recognize PS and positively or negatively regulate the phagocytosis of apoptotic cells. CD300a and CD300f have been reported to negatively regulate phagocytosis, ${ }^{17,19}$ which correlates with the fact that they contain ITIM motifs in their cytoplasmic tails that can bind phosphatases. ${ }^{16,19,26}$ In agreement with this findings, blocking of CD300a and PS interactions induces increased inflammatory cytokine production from mast cells, ${ }^{26}$ and CD300f deficiency leads to a predispostion to autoimmune disease development, ${ }^{19}$ as well as an exacerbation in the allergic responses and autoimmune diseases. ${ }^{19,25,33,34}$ In contrast, our data characterize CD300b as a positive regulator of phagocytosis, through its association with DAP12. The exact role of $\mathrm{CD} 300 \mathrm{~b}$ in efferocytosis remains to be elucidated, but the existing data suggest that CD300b is involved in the regulation of some immune responses. For instance, CD300b deficient mice are protected from ischemia/ reperfusion injury $(\mathrm{IRI}) .{ }^{23}$ As the blockade of $\mathrm{PS}$ exposure on the cell surface by diannexin is able to improve kidney and liver IRI, ${ }^{35,36}$ CD300b could be involved in $|R|$ via PS recognition. In addition, CD300b-deficient mice are resistant to endotoxin and sepsis models. ${ }^{22,37}$ The excessive inflammatory responses induced by microbes leads to apoptosis of lymphocytes and epithelial cells, ${ }^{37}$ and hence CD300b, which recognizes these apoptotic cells, could be involved in the pathology of sepsis. Thus, CD300b likely contributes to the development of these pathologies, and CD300 family members appear to both positively and negatively regulate immune responses.

PS receptors regulate the immune response and cellular homeostasis through efferocytosis. Receptor protein tyrosine kinase TAM family members, Tyro3, AxI and MerTK, associate with apoptotic cells by binding to PS through bridging molecules, like Gas6 or Protein S, resulting in a suppression of inflammatory responses. ${ }^{15}$ Lack of TAM family members has been associated with autoimmune diseases,

Figure 3 Indirect binding of TIM1 to CD300b is mediated by phosphatidylserine. (a) EV- or TIM1-transduced Ba/F3 cells were stained with Annexin V-APC, 7-AAD and anti-TIM1-PE antibody. The contour plots show TIM1-PE and Annexin V-APC staining of the 7-AAD' cell populations. (b) EV-or TIM1-transduced Ba/F3 cells were cocultured with CD300b- or NITR-Fc proteins and stained with 7-AAD, anti-TIM1-PE and anti-human IgG Fc $\gamma$-FITC antibodies. Graphs show binding of the Fc-fused proteins and TIM1 to the 7-AAD- cell populations. (c). EV- or TIM1-transduced Ba/F3 cells were preincubated with unlabeled Annexin V $(10 \mu \mathrm{g})$ and then cells were stained with Annexin V-APC (left panel) or the indicated Fc-fused proteins (right panel), followed by anti-human IgG Fc $\gamma$-specific antibody. (d) EV- or TIM1-transduced Ba/F3 cells were incubated with biotin-labeled PC- or PS-coated liposomes, stained with streptavidin-APC and liposome binding was detected by flow cytometry. (e) EV-or TIM1-transduced Ba/F3 cells were cultured with PC- or PS-coated liposomes and incubated with CD300b- or NITR-Fc proteins. Cells were stained with anti-human IgG Fc $\gamma$-FITC antibody and analyzed by flow cytometry. The bar graph shows the MFI values + S.E.M. of CD300b-Fc bound to the cell surface; the data are from three independent experiments. (f) Recombinant murine TIM1 protein was immobilized on CM5 biosensor, and binding of CD300b-Fc or NITR-Fc proteins was analyzed by SPR, as in Figure 1 . Binding was initiated at time $=60 \mathrm{~s}$ and the washout (dissociation phase) was begun at $t=240 \mathrm{~s}$. (g) Binding of CD300b-Fc or NITR-Fc proteins to TIM1 using a two-step reaction. TIM1 was immobilized on the CM5 sensor chip, and then PC- or PS-coated liposomes were flown over the TIM1 coated chip (first step; 1st). Next, CD300b-Fc or NITR-Fc proteins were injected (second step, 2nd), and the binding to TIM1 was analyzed by SPR. The top panel illustrates a representative result of the SPR analysis, whereas the bottom graph shows the changes in resonance units (RU) following the addition of CD300b-Fc or NITR-Fc (the second step of the reaction). All results are a representative of three independent experiments with a mean + S.E.M.; ${ }^{* \star} P<0.01$. ${ }^{* \star} P<0.001$ (Student's $t$-test) 
a

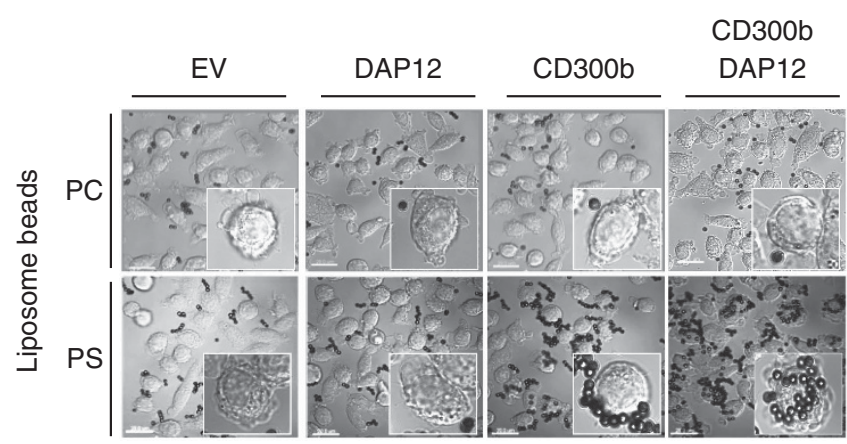

b

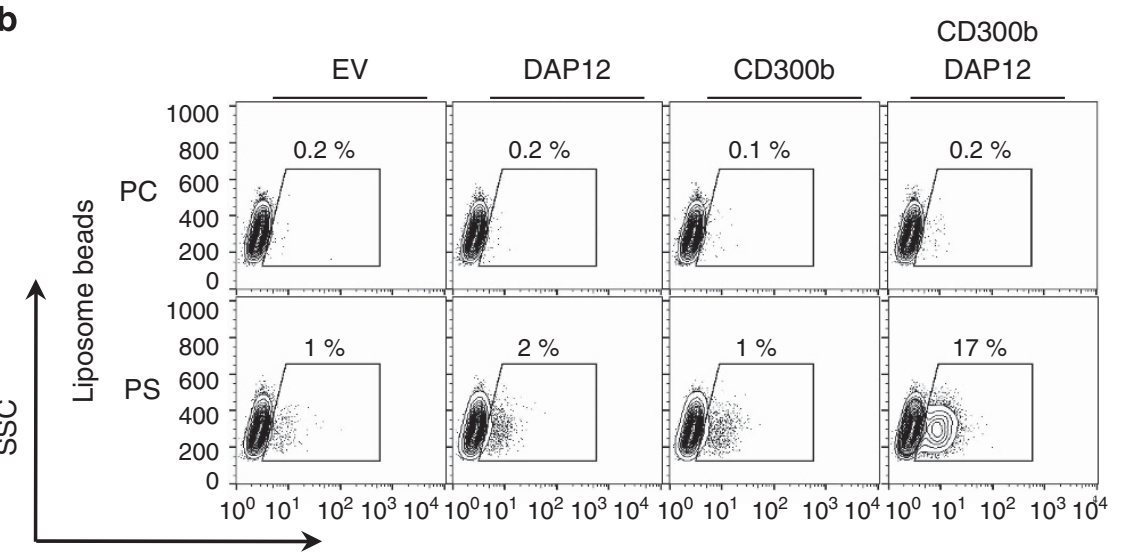

PKH67

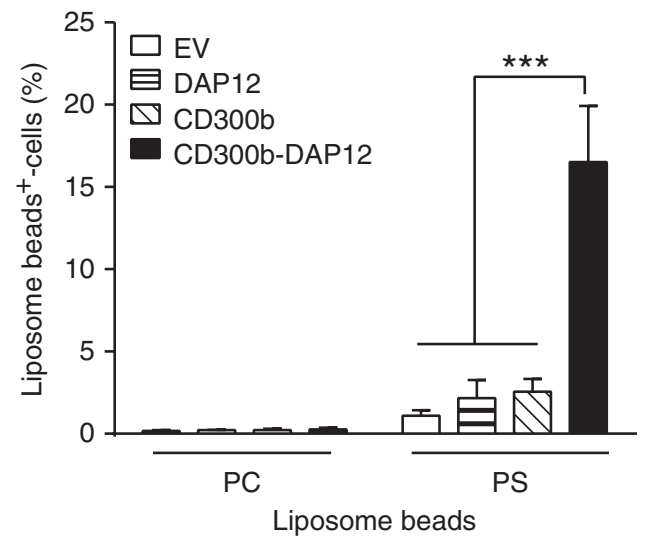

Figure 4 CD300b-mediated engulfment of phosphatidylserine liposome-coated beads requires the co-expression of DAP12. (a) L929 cells transduced with the indicated constructs were incubated with PC- or PS-coated liposome beads for $20 \mathrm{~min}$ at $37^{\circ} \mathrm{C}$. The cells were fixed, and imaged by microscopy. The DIC images show cells and liposome-coated beads; the scale bar is $20 \mu \mathrm{m}$. The inserts show a close-up of a single cell. (b) PKH67-labeled L929 cells transduced with the indicated constructs were incubated with PC- or PS-coated liposome beads for $30 \mathrm{~min}$. After homogenization of the cells, the beads were harvested, and their phagocytosis was determined by analysis of the percentage of $\mathrm{PKH}_{67}{ }^{+}$-beads using flow cytometry. The beads that became fluorescent were regarded as the engulfed beads, as they acquired the fluorescence due to their encapsulation within $\mathrm{PKH} 67^{+}$-cell membranes (phagosomes). The contour plot indicates the gating strategy, and illustrates a representative result (upper panel). The bar graph (lower panel) shows the quantification of the percentage of engulfed liposome-coated beads. Data represent mean + S.E.M.; ${ }^{* *} P<0.001$ (ANOVA)

like SLE. ${ }^{38}$ TIM4 does not have any signaling motif in its cytoplasmic domain but promotes engulfment of activated antigen-specific T cells, ${ }^{39}$ as well as apoptotic cells, ${ }^{11,12}$ via PS recognition by utilizing $\beta 1$ integrins as co-receptors. ${ }^{40}$ Deficiency of TIM4 expression is linked to reduced tolerance, ${ }^{39}$ enhanced tumor immunity ${ }^{41}$ and the development of autoimmunity. ${ }^{12}$ Another PS receptor, BAI1, mediates engulfment of apoptotic cells, but unlike TIM4, directly activates downstream signaling events. ${ }^{13}$ Furthermore, BAl1 promotes fusion of healthy myoblasts ${ }^{42}$ and is also important for Sertolicell-mediated engulfment. ${ }^{43}$ Stabilin2-mediated uptake of apoptotic cells requires the adaptor molecule, GULP. ${ }^{44}$ Our findings demonstrate that CD300b alone can not only tether apoptotic cells, but can mediate efferocytosis when associated with the adaptor molecule, DAP12. Importantly, our results showed that CD300b promotes phagocytosis of apoptotic cells, 

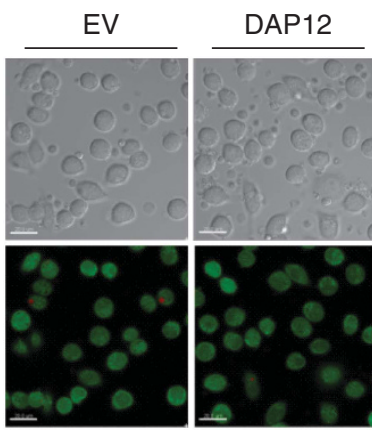

b



pHrodo ${ }^{+}$-cells

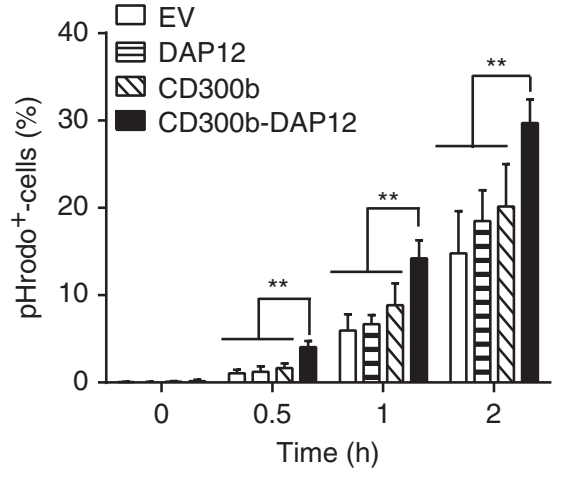

C

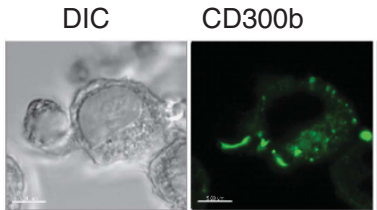

Phalloidin

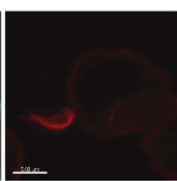

CD300b

DAP12

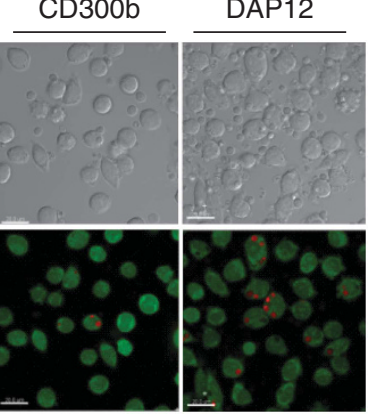

CD300b

DAP12

。

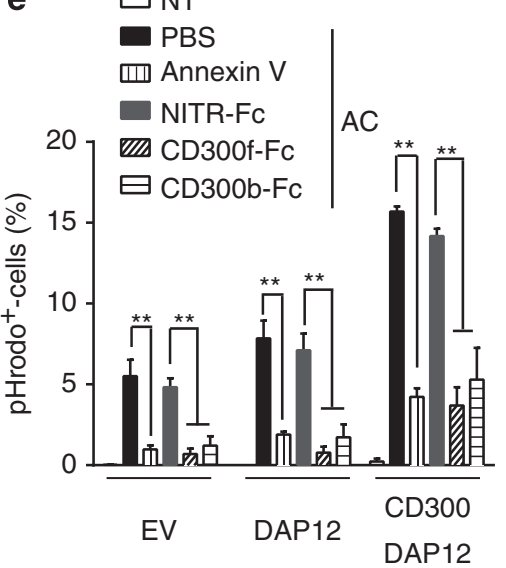

f

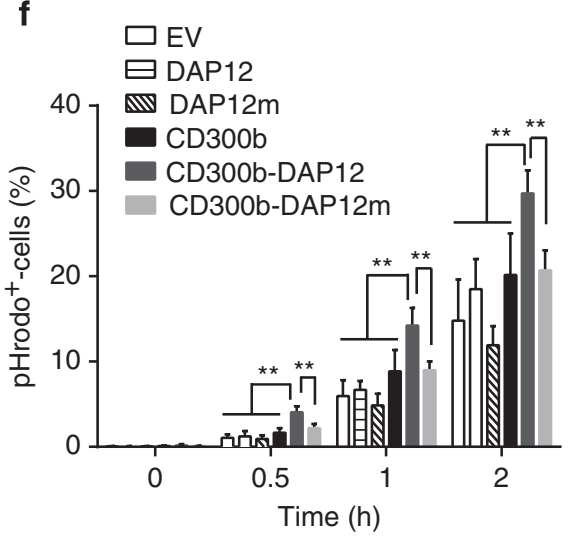

Figure 5 CD300b promotes efferocytosis in a DAP12-dependent manner. (a) EV-, DAP12-, CD300b-and CD300b-DAP12-expressing L929 cells were labeled with CFSE, mixed with pHrodo-labeled apoptotic cells (AC) and the percentage of engulfment of (pHrodo ${ }^{+}$) cells was analyzed by confocal microscopy (a) and flow cytometry (b). (a) CFSE-labeled L929 cells are shown green, while engulfed pHrodo ${ }^{+}$-labeled apoptotic cells are displayed in red (lower panel); the DIC images are shown in the upper panel. Scale bars, $20 \mu \mathrm{m}$. (b) The contour plots (upper panel) illustrate the gating strategy whereas the quantification of the percentage of pHrodo ${ }^{+}$cells is summarized in the bar graph (bottom panel; error bars represent S.E.M.). (c) L929 cells expressing Myc-tagged CD300b were cultured in glass-bottom dishes, and incubated with TFL4-labeled apoptotic cells (AC, blue). The cells were then fixed, washed and stained using anti-cMyc antibody, followed by Alexa647-conjugated secondary antibody (green). F-actin was stained using phalloidin-Alexa568 (red). Scale bars, $5 \mu \mathrm{m}$. (d) EV-, DAP12- and CD300b-DAP12-expressing L929 cells were cultured in the presence or absence of PC-, PE- or PS-coated liposomes $(10 \mu \mathrm{M})$ and then mixed at a 1:4 ratio with pHrodo-labeled apoptotic cells $(\mathrm{AC})$ for 60 min. Percentage of cells containing apoptotic thymocytes (pHrodo ${ }^{+}$-cells) was analyzed by flow cytometry, as described in b. (e) pHrodo-labeled apoptotic cells (AC) were cultured in the presence or absence of $50 \mu \mathrm{g} / \mathrm{ml}$ Annexin V, CD300f-Fc, CD300b-Fc or NITR-Fc proteins and then incubated with EV-, DAP12- and CD300b-DAP12-expressing L929 cells at a 4:1 ratio for 60 min. Percentage of pHrodo $^{+}$-cells was analyzed by flow cytometry, as in b. (f) EV-, DAP12-, DAP12m-, CD300b-DAP12-, CD300b-DAP12m-expressing L929 cells were mixed with pHrodolabeled apoptotic cells (AC) at a 1:4 ratio, and the percentage of pHrodo ${ }^{+}$-cells was analyzed by flow cytometry, as in $\mathbf{b}$. All data shown are derived from three independent experiments, and the bar graphs shown in $\mathbf{b}$ and $\mathbf{d}$-f represent mean + S.E.M.; ${ }^{* *} P<0.01$ (Student's $t$-test) 
a

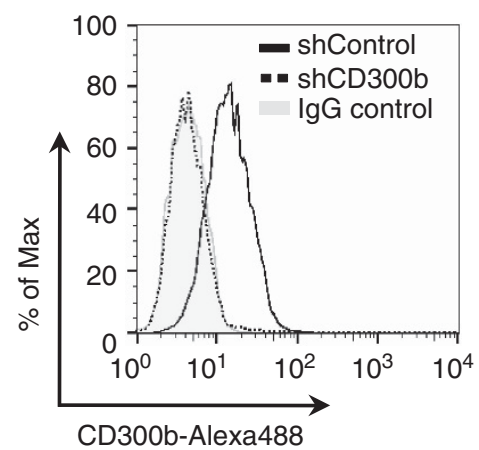

b

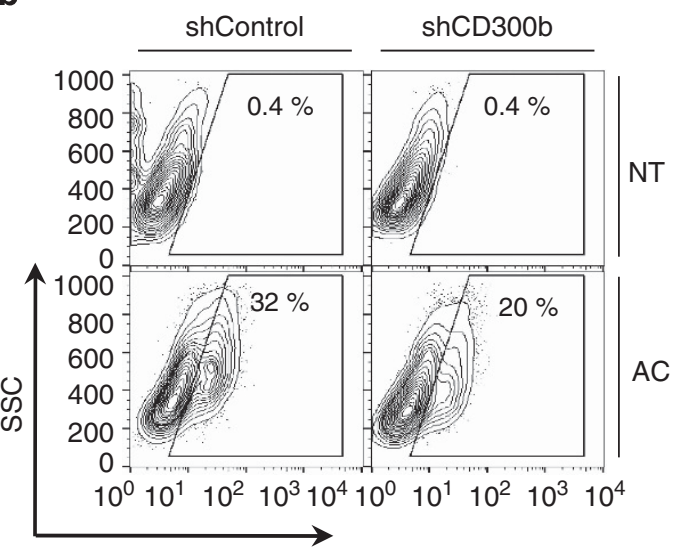

pHrodo ${ }^{+}$-cells

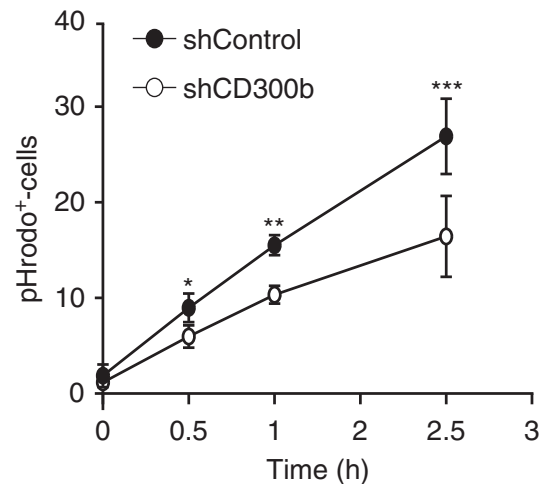

C

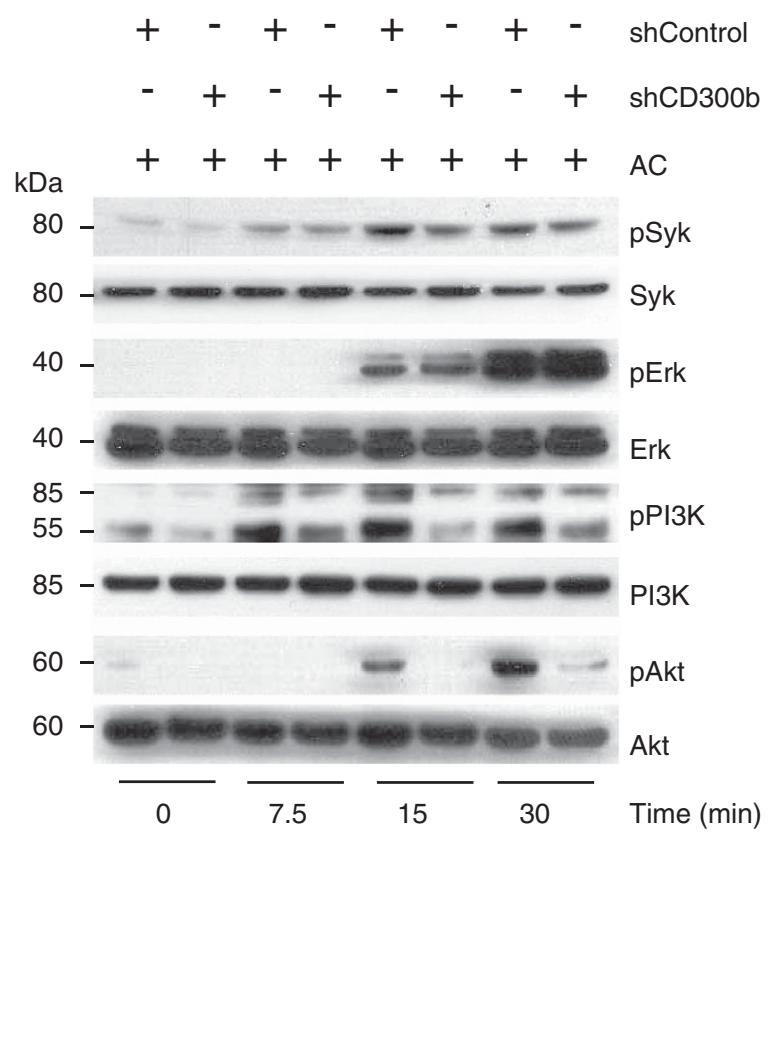

Figure 6 CD300b regulates macrophage-mediated phagocytosis of apoptotic cells. (a) J774.1 cells transduced with short hairpin scramble control RNA (shControl), or shRNA targeting CD300b (shCD300b) were stained with Alexa488-labeled anti-CD300b antibody. The efficiency of silencing was assessed by monitoring CD300b cell surface expression via flow cytometry. (b) Control or CD300b-silenced J774.1 cells were mixed with pHrodo-labeled apoptotic cells (AC; 1:4 ratio), and the percentage of

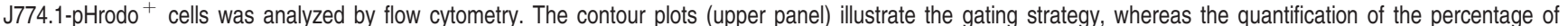
J774.1-pHrodo ${ }^{+}$-cells from three independent experiments is summarized in the bar graph (bottom panel; error bars represent S.E.M.); ${ }^{*} P<0.05$, ${ }^{* *} P<0.01$ (Student's $t$-test). (c) Control or CD300b-silenced J774.1 cells were incubated with or without apoptotic cells (AC) for the indicated length of time, and the activation of DAP12-dependent signaling pathways was assessed by western blot analysis with anti-pSyk, anti-pAkt, anti-pErk, anti-pPI3K antibodies. Immunoblot using anti-Syk, anti-Erk, anti-Akt and antiPI3K-antibodies served as loading controls

without the presence of other PS receptors. However, different PS receptors, like MerTK and TIM4, ${ }^{45}$ or $\alpha \mathrm{v} \beta 3$ and CD36, ${ }^{46}$ can cooperate to enhance efferocytosis. ${ }^{45}$ Therefore, it is likely that efferocytosis mediated by members of the CD300 family, such as CD300b, can be further enhanced through the cooperation with other PS receptors, like TIM1 or TIM4, suggesting a mechanism of corporative engagement of PS receptors for efficient apoptotic cell clearance.
CD300b has a short intracellular domain without any known signaling motifs; ${ }^{21}$ however it contains a positively charged lysine residue within its transmembrane segment, which could promote pairing with an appropriate adaptor molecule. ${ }^{21}$ Both DAP10 and DAP12 have a negatively charged aspartic acid residue within their transmembrane domains making them suitable for pairing with CD300b. The fact that CD300b associates preferentially with DAP12 over DAP10 ${ }^{21}$ agrees 
with our finding that DAP12 is more efficient in promoting CD300b-mediated efferocytosis. We further found that pairing with DAP12 was necessary for CD300b to promote apoptotic cell engulfment, but is not necessary for apoptotic cell attachment. Upon receptor ligation, DAP12 has been shown to initiate downstream signaling through phosphorylation of the tyrosine residue within its ITAM motif. ${ }^{28}$ This data are further supported by our observation that a tyrosine to phenylalanine mutation within DAP12 (DAP12m) failed to support CD300b-mediated efferocytosis. Anti-CD300b antibody cross-linking promotes Syk, Erk and Akt phosphorylation via DAP12. ${ }^{21}$ We found that, while Syk, Erk, PI3K and Akt phosphorylation in macrophages is induced upon apoptotic cell recognition, only Syk, PI3K and Akt phosphorylation are markedly impaired in CD300b-silenced macrophages. This indicates that, in response to apoptotic cell recognition, CD300b regulates $\mathrm{PI} 3 \mathrm{~K}$ activation via interaction with DAP12 and Syk, and that the PI3K pathway is the major signaling pathway used in CD300b-mediated phagocytosis. It has been reported that Erk signaling also regulates the engulfment of apoptotic cells, ${ }^{47,48}$ and DAP12 has been shown to activate the Erk signaling. ${ }^{28}$ Although we observed Erk activation following cross-linking of CD300b, we did not find any substantial change in Erk activation in CD300b silenced cells. This result indicates that CD300b is not directly involved in activation of the Erk signaling pathway and suggests that other PS receptors, such as class B scavenger receptor type I or ATP-binding cassette transporter A7, 47,48 could provide signaling cues that lead to the activation for Erk signaling pathway, following the recognition of apoptotic cells.

In summary, our report demonstrates that CD300b is able to recognize PS exposed on the apoptotic cell surface, and transduces the signals through the DAP12Syk-PI3K-AKT pathway, thereby promoting apoptotic cell phagocytosis.

\section{Materials and Methods \\ Cells and antibodies. L929, HEK293T or J774.1 cells were cultured in DMEM medium with $10 \%$ FBS. Anti-mouse CD300b antibody and isotype control goat IgG were from R\&D (Minneapolis, MN, USA), and were labeled with Alexa488 using the Alexa488 Monoclonal Antibody Labeling Kit (Invitrogen, Grand Island, NY, USA), according to the manufacturer's instructions. Anti-cMyc tag (9B11), anti-Akt, anti-pAkt (D9E), anti-pSyk, anti-Syk, anti-pErk (3A7), anti-Erk, anti-pPI3K (Y199) and p85 antibodies were from Cell Signaling Technology (Danvers, MA, USA). Anti-cMyc antibody (9E10), and HRP-conjugated secondary antibodies (goat anti-rabbit and goat anti-mouse) were from Santa Cruz Biotechnology (Santa Cruz, CA, USA). Anti-mouse CD16/CD32 antibody (93) was from eBioscience (San Diego, CA, USA). Anti-human IgG Fcy fragment specific antibody was from Jackson ImmunoResearch (West Grove, PA, USA).}

DNA reagents. For the CD300b, DAP10 and DAP12 lentivirus expression constructs, the PCR products were cloned into the pCDH-EF1-T2A-puro (pCDH) vector (System Biosciences, Mountain View, CA, USA), using the EcoRI and Notl sites; cMyc tag was added at the C-terminus of CD300b constructs, unless stated otherwise. Tyrosine to phenylalanine substitution in the immunoreceptor tyrosinebased activation (ITAM) motif within the cytoplasmic domain of DAP12 (DAP12m) was generated by site-directed mutagenesis using the QuickChange Mutagenesis Kit (Stratagene, Santa Clara, CA, USA), according to the manufacturer's instructions. The constructs for human IgG1 Fc portion fused to CD300b (CD300b$\mathrm{Fc}), \mathrm{CD} 300 \mathrm{f}(\mathrm{CD} 300 \mathrm{f}-\mathrm{Fc})$ or the control protein, NITR (NITR-Fc), extracellular domains in a pcDNA backbone were kindly supplied by Dr. John P. Cannon. ${ }^{24}$ The constructs for mouse TIM1 or TIM4 in pMX retroviral vector were kindly supplied by Dr. Shigekazu Nagata. ${ }^{12}$ Lentiviral short hairpin (sh)RNA vector against
CD300b and control shRNA vector were purchased from Santa Cruz Biotechnology.

Cell transfection and infection. HEK293T cells were transfected using Lipofectamine 2000 (Invitrogen). Lentivirus particles were generated by cotransfection of HEK293T cells with PCDH-puro expression or shRNA vectors, and psPAX2 and pMD2G helper plasmids. The infection of L929, J774.1 and Ba/F3 cells was carried out by incubating the cells with lentivirus for $24 \mathrm{~h}$ at $37^{\circ} \mathrm{C}$, in the presence of $6 \mu \mathrm{g} / \mathrm{ml}$ protamine sulfate. Selection with puromycin $(20 \mu \mathrm{g} / \mathrm{ml}$ for L929 and $6 \mu \mathrm{g} / \mathrm{ml}$ for $\mathrm{J774}$ cells) was started $48 \mathrm{~h}$ after infection. L929 cells expressing equivalent amounts of CD300b were cloned using the limiting dilution method.

Chimeric proteins. HEK293T cells were transfected with pcDNA3.0 plasmids encoding CD300b-Fc, CD300f-Fc and NITR-Fc constructs using Lipofectamine 2000, as described above. Forty-eight hours after transfection, the culture supernatant, containing the chimeric proteins, was harvested and the Fc-fusion proteins were purified using protein A-Sepharose Fast Flow columns (Amersham Biosciences, Arlington Heights, IL, USA). Protein expression and purity were verified by Colloidal Coomassie Blue staining and SDS-polyacrylamide gel electrophoresis followed by western blot analysis. His-tagged TIM1 protein was obtained from R\&D systems.

Preparation of phospholipid-containing liposomes and liposomecoated beads. 1-palmitoyl-2-oleoyl-sn-glycero-3-phosphocholine (POPC), 1-palmitoyl-2-oleoyl-sn-glycero-3-phosphoethanolamine (POPE), 1-palmitoyl-2-oleoylsn-glycero-3-phospho-L-serine (POPS), 1,2-dioleoyl-sn-glycero-3-phosphocholine (DOPC), 1,2-dioleoyl-sn-glycero-3-phosphoserine (DOPS), 1,2-dioleoyl-sn-glycero3-phosphoethanolamine (DOPE) were from Avanti Polar Lipids (Alabaster, AL, USA). N-(biotinoyl)-1,2-dihexadecanoyl-sn-glycero-3-phosphoethanolamine, triethylammonium salt (biotin-labeled DHPE) was from Invitrogen. Liposomes were prepared by completely evaporating the chloroform from the desired phospholipid mixture with argon gas. Large multilamellar vesicles were formed by swirling in degassed PBS ( $\mathrm{pH}$ 7.4). Unilamellar vesicles were prepared by extrusion through filters with $100 \mathrm{~nm}$ pore size, using Avanti Mini-Extruder (Avanti Polar Lipids). Liposome composition was $80 \%$ DOPC: $20 \%$ of POPC (PC), POPS (PS) or POPE (PE). The size of the liposomes size was verified as approximately $100 \mathrm{~nm}$ by Dynamic Light Scattering (DLS). ${ }^{49}$ For experiments using liposomalcoated beads, the lipid composition was 49\% DOPC: $50 \%$ DOPS: $1 \%$ biotinlabeled DOPE. M-280 streptavidin-coated Dynabeads (Invitrogen) were first pre-incubated in PBS with $1 \%$ BSA (Sigma, St. Louis, MO, USA) at $37^{\circ} \mathrm{C}$ for $2 \mathrm{~h}$, then mixed with liposomes for $2 \mathrm{~h}$ at $37^{\circ} \mathrm{C}$ in degassed PBS with $1 \% \mathrm{BSA}$, followed by two washing steps and re-suspension in the same buffer. The presence of PS on the beads was confirmed by staining with Annexin V-APC and flow cytometry analysis. Liposomes and liposome-coated beads were stored in argon gas to prevent lipid oxidization.

Phagocytosis assay. Apoptotic thymocytes were generated by incubating cells at $37^{\circ} \mathrm{C}$ for $6 \mathrm{~h}$ with $10 \mu \mathrm{M}$ dexamethasone (Sigma), at which time at least $80 \%$ of the cells were Annexin $\mathrm{V}^{+}$. The apoptotic cells were labeled with TFL-4 (Oncoimmunin Inc., Gaithersburg, MD, USA) or pHrodo (Invitrogen), and added to the phagocytes at a ratio 1:4 or 1:15 as indicated in the text. After incubation, the phagocytes were washed, treated with trypsin/EDTA, fixed and analyzed by flow cytometry, or visualized by LSM710 laser-scanning confocal microscopy (Carl Zeiss, Thornwood, NY, USA). For experiments using pHrodo-labeled thymocytes for microscopic or flow cytometry analyses, cells were washed and resuspended in basic buffer ( $\mathrm{pH} \mathrm{8.8)} \mathrm{to} \mathrm{quench} \mathrm{the} \mathrm{fluorescence} \mathrm{of} \mathrm{non-phagocytosed} \mathrm{pHrodo-}$ labeled apoptotic cells. In blocking experiments involving Fc-fused proteins or Annexin $V$ as a blocking agent, apoptotic cells were preincubated with $50 \mu \mathrm{g} / \mathrm{ml}$ of protein for $10 \mathrm{~min}$ at $37^{\circ} \mathrm{C}$ before adding to the phagocytes. In experiments involving liposomes as a blocking agent, L929 cells were preincubated with $10 \mu \mathrm{M}$ liposomes for $10 \mathrm{~min}$ at $37^{\circ} \mathrm{C}$ before adding pHrodo-labeled apoptotic cells. In experiments using phospholipid-coated liposome beads, the liposome beads were added to phagocytes for $15 \mathrm{~min}$ at $37^{\circ} \mathrm{C}$, followed by three washes with PBS, fixation and analysis using confocal microscopy. F-actin was visualized using Alexa568-conjugated phalloidin. For blocking experiments using anti-CD300b antibody, primary macrophages were isolated from the peritoneal cavity and cultured for $24 \mathrm{~h}$ at $37^{\circ} \mathrm{C}$. Macrophages $\left(2 \times 10^{5}\right)$ were pre-treated with $25 \mu \mathrm{g}$ anti-CD300b or anti-IgG-control antibodies for $15 \mathrm{~min}$, cocultured with 
pHrodo-labeled apoptotic thymocytes at a ratio of 1:4 for additional $30 \mathrm{~min}$ at $37^{\circ} \mathrm{C}$. Cells were harvested, washed with PBS containing $2 \% \mathrm{FBS}$ and stained with anti-F4/80-APC. Percentage of efferocytosis was assessed by flow cytometry. L929 cells stained with PKH67 (Sigma) were incubated with PC- or PS-coated liposome beads for $30 \mathrm{~min}$. Cells were collected and suspended in ice-cold homogenization buffer $(0.25 \mathrm{M}$ sucrose, $10 \mathrm{mM}$ HEPES ( $\mathrm{pH} 7.4)$, protease inhibitor cocktail in PBS), and then homogenized by passage through a bent 27 1/2 gauge needle 20 times. The disruption of cell integrity was confirmed by microscopic inspection. Beads were harvested from the homogenates using a Dynal magnet, washed three times with cold PBS and analyzed for PKH67 fluorescence by flow cytometry. The engulfed beads showed higher PKH67 fluorescence due to acquisition of the cell membrane (phagosome).

Flow cytometry. Alexa488-conjugated anti-CD300b antibody was used to examine the expression of CD300b on the cell surface. Alexa488-conjugated goat IgG was used as the control. PE-conjugated anti-TIM1 or anti-TIM4 antibodies were used to examine the expression of TIM1 or TIM4 by Ba/F3 cells. Cells were stained with the antibody for $30 \mathrm{~min}$ at $4{ }^{\circ} \mathrm{C}$, washed and analyzed by flow cytometry.

For receptor-binding analysis, $0.5 \times 10^{6}$ apoptotic thymocytes or Ba/F3 cells were incubated with $5 \mu \mathrm{g} / \mathrm{ml}$ anti-mouse $\mathrm{CD} 16 / \mathrm{CD} 32$ to block Fc receptors and then incubated with 0.05 or $0.1 \mu \mathrm{g} / \mathrm{ml}$ chimeric proteins, CD300b-Fc or NITR-Fc, on ice for $40 \mathrm{~min}$, followed by staining with FITC or APC-conjugated anti-human IgG Fc $\gamma$ fragment specific antibodies (Jackson ImmunoResearch) for $15 \mathrm{~min}$. Cells were washed with $2 \% \mathrm{FBS}$ in PBS and analyzed by flow cytometry. In the case of $\mathrm{Ba} / \mathrm{F} 3$ cells, reactions were stained with 7-AAD (BD Bioscience, San Jose, CA, USA) to exclude dead cells. In experiments involving recombinant unlabeled Annexin $V$ as a blocking agent, apoptotic cells or Ba/F3 cells were preincubated with Annexin $V$ for 20 min on ice before incubation with Fc-chimeric proteins or Annexin V-APC. In experiments using liposomes as a blocking agent, liposomes were incubated with Fc-chimeric proteins for $10 \mathrm{~min}$ at RT and then the samples were incubated with apoptotic cells. To detect CD300b binding to liposomes attached to cells, $\mathrm{Ba} / \mathrm{F} 3$ cells were incubated with liposomes for $30 \mathrm{~min}$ on ice and then the cells were incubated with $\mathrm{Fc}$-chimeric proteins. Streptavidin-APC was used to detect liposomes bound to $\mathrm{Ba} / \mathrm{F} 3$ cells.

For phagocytosis analysis, the samples were prepared as described above. The phagocytes and apoptotic cells were distinguished by forward and side scatter characteristics. The appearance of pHrodo fluorescence in the phagocyte population was monitored as an indicator of the apoptotic cell engulfment: the phagocyte cell population characterized by high fluorescence of (i.e., pHrodo ${ }^{\text {high }}$ ) was regarded as the cells that engulfed the apoptotic cells. The data acquisition and analysis was done using FACSort flow cytometer (BD Bioscience) with Cell Quest software (version 3.3), and analyzed using FlowJo (v.7.6; Tree Star, Ashland, OR, USA).

Western blot analysis. J774 cells $\left(1 \times 10^{6}\right)$ were incubated with $4 \times 10^{6}$ apoptotic thymocytes for $10 \mathrm{~min}$ at $4^{\circ} \mathrm{C}$, and then transferred to $37^{\circ} \mathrm{C}$ for the indicated time. Extracts were prepared in lysis buffer containing $50 \mathrm{mM}$ Tris- $\mathrm{HCl}$ (pH 7.4), $150 \mathrm{mM} \mathrm{NaCl}, 5 \mathrm{mM}$ EDTA, $1 \% \mathrm{NP}-40,0.5 \%$ sodium deoxycholate, and protease and phosphatase inhibitors cocktail (Sigma). Cellular debris was removed by centrifugation at $10000 \times g$ for $15 \mathrm{~min}$ at $4^{\circ} \mathrm{C}$. Cell lysates were resolved by SDS-PAGE, and transferred onto PVDF membranes (Millipore, Billerica, MA, USA). Membranes were blocked with $5 \%$ BSA (Sigma) in TBST (20 $\mathrm{mM}$ Tris pH 7.4, $150 \mathrm{mM} \mathrm{NaCl}, 0.05 \%$ Tween-20) for $1 \mathrm{~h}$, and then probed with the antibodies as indicated in the text. HRP-conjugated secondary antibodies were used and immunoblots were developed with West Pico SuperSignal kit (Pierce, Rockford, IL, USA) and visualized on Hyperfilm (Amersham Biosciences).

Confocal microscopy. For the cellular location of CD300b during phagocytosis, cells expressing CD300b-cMyc were incubated with or without TFL4-labeled apoptotic cells. Cells were plated on number 1.5 glass dishes (MatTek Corporation, Ashland, MA, USA), fixed with $4 \%$ paraformaldehyde in PBS for $15 \mathrm{~min}$ at room temperature, and then visualized by confocal microscopy. To label F-actin, cells were fixed as described above, permeabilized with $0.4 \%$ saponin in PBS for 15 min and stained with Alexa568-conjugated phalloidin (1:200 dilution) for $1 \mathrm{~h}$. In the cases of indirect immunofluorescence staining, cells were fixed and permeabilized as described above, and then blocked with $10 \%$ BSA in $\mathrm{PBS}$, followed by incubation with the primary antibody for $1 \mathrm{~h}$ at room temperature. Anti-cMyc antibody was used at 1:2000. Finally, samples were incubated for $1 \mathrm{~h}$ with goat anti-mouse antibodies (1:500 dilution) conjugated with Alexa488, 569 or 647, as indicated in the figure legends. Cells were mounted in ProLong Gold medium (Invitrogen), and visualized by a LSM 710 laser scanning confocal microscope (Zeiss) with a $63 \times$ Zeiss Plan-Apochromat objective, and analyzed using Imaris software (v 7.4; Bitplane, South Windsor, CT, USA).

SPR. The interaction of Fc-chimeric proteins with different phospholipids was measured using the BIAcore 2000 system (Biacore Inc., Pittsburgh, PA, USA) according to our previous report. ${ }^{17}$ For liposomes and Fc-fused protein interactions, $1 \mathrm{mM}$ liposomes were captured on an L1 sensor chip, followed by exposure to Fc-fused proteins at $10 \mu \mathrm{g} / \mathrm{ml}$. For measuring the interaction between TIM1 and CD300b, TIM1 was coupled to a CM5 sensor chip using standard NHSEDC coupling. Then, the different Fc-fused proteins were injected at $10 \mu \mathrm{g} / \mathrm{ml}$ to analyze their binding to TIM1. For measuring the binding of CD300b-Fc to TIM1 plus various phospholipid-containing liposomes, TIM1 was immobilized on a CM5 sensor; followed by the injection of liposomes $(300 \mu \mathrm{M})$. After that, the binding of Fc-fused proteins was determined.

RNA isolation and quantitative real-time RT-PCR. Total RNA was isolated using the RNAquous-4PCR kit (Ambion, Grand Island, NY, USA) following the manufacturer's instructions. CDNA was synthesized with Qscript cDNA synthesis kit (Quanta Biosciences, Gaithersburg, MD, USA) and quantitative realtime PCR (qRT-PCR) was performed as previously described. ${ }^{50}$ Oligonucleotide primers for amplifying murine CD300b, CD300f, MerTK, Axl, BAl1, Stabilin 2 and $G A P D H$ were purchased from Qiagen. Fold change of expression (relative copy number $(\mathrm{RCN})$ ) of selected genes was normalized by the expression of the housekeeping gene, GAPDH and calculated with the equation: $\mathrm{RCN}=\mathrm{E}^{-\Delta \mathrm{Ct}}$, where $\mathrm{E}=$ efficiency of $\mathrm{PCR}$, and $\mathrm{Ct}=\mathrm{Ct}$ target $-\mathrm{Ct} \mathrm{GAPDH}$. Melting curve analyses were performed at the end of each run to ensure that only one product was amplified.

Statistical analysis. The statistical significance was assessed using ANOVA with Bonferroni post test, or by the two-tailed unpaired Student's t-test (GraphPad Prism Software, version 6.0). Data are presented as mean + SEM, unless stated otherwise. Alpha level was set to 0.05 .

\section{Conflict of Interest}

The authors declare no conflict of interest.

Acknowledgements. We thank Dr. John P. Cannon for the CD300b-Fc and NITR-Fc vectors. We thank Dr. Shigekazu Nagata for the pMX-puro TIM1 and TIM4 vectors, and Dr. Joseph Brzostowski for the technical help with the microscopy. We thank Ms. Lisa Boyd for SPR technical assistance, and Dr. Patrick H. Brown for DLS. We thank Dr. Aleksandra Gil-Krzewska, and Venkateswara Simhadri for their critical comments. This study was supported by the intramural programs of the National Institute of Allergy and Infectious Diseases.

1. Erwig LP, Henson PM. Clearance of apoptotic cells by phagocytes. Cell Death Differ 2008; 15: 243-250.

2. Flannagan RS, Jaumouille V, Grinstein S. The cell biology of phagocytosis. Annu Rev Pathol 2012; 7: 61-98.

3. Savill J, Fadok V. Corpse clearance defines the meaning of cell death. Nature 2000; 407: 784-788.

4. Stuart LM, Ezekowitz RA. Phagocytosis and comparative innate immunity: Learning on the fly. Nat Rev Immunol 2008; 8: 131-141.

5. Elliott MR, Ravichandran KS. Clearance of apoptotic cells: Implications in health and disease. J Cell Biol 2010; 189: 1059-1070.

6. Ferguson TA, Choi J, Green DR. Armed response: how dying cells influence T-cell functions. Immunol Rev 2011; 241: 77-88.

7. Lauber K, Blumenthal SG, Waibel M, Wesselborg S. Clearance of apoptotic cells: getting rid of the corpses. Mol Cell 2004; 14: 277-287.

8. Silva MT. Secondary necrosis: the natural outcome of the complete apoptotic program. FEBS Lett 2010; 584: 4491-4499.

9. Park SY, Jung MY, Kim HJ, Lee SJ, Kim SY, Lee BH et al. Rapid cell corpse clearance by Stabilin-2, a membrane phosphatidylserine receptor. Cell Death Differ 2008; 15 : 192-201.

10. Park SY, Jung MY, Lee SJ, Kang KB, Gratchev A, Riabov V et al. Stabilin-1 mediates phosphatidylserine-dependent clearance of cell corpses in alternatively activated macrophages. J Cell Sci 2009; 122: 3365-3373. 
11. Kobayashi N, Karisola P, Pena-Cruz V, Dorfman DM, Jinushi M, Umetsu SE et al. Tim1 and Tim4 glycoproteins bind phosphatidylserine and mediate uptake of apoptotic cells. Immunity 2007; 27: 927-940.

12. Miyanishi M, Tada K, Koike M, Uchiyama Y, Kitamura T, Nagata S. Identification of Tim4 as a phosphatidylserine receptor. Nature 2007; 450: 435-439.

13. Park D, Tosello-Trampont AC, Elliott MR, Lu M, Haney LB, Ma Z et al. Bai1 is an engulfment receptor for apoptotic cells upstream of the Elmo/Dock180/Rac module. Nature 2007; 450: 430-434

14. Hanayama R, Tanaka M, Miwa K, Shinohara A, Iwamatsu A, Nagata S. Identification of a factor that links apoptotic cells to phagocytes. Nature 2002; 417: 182-187.

15. Lemke G, Rothlin CV. Immunobiology of the Tam receptors. Nat Rev Immunol 2008; 8 : 327-336.

16. Nakahashi-Oda C, Tahara-Hanaoka S, Honda S, Shibuya K, Shibuya A. Identification of phosphatidylserine as a ligand for the CD300a immunoreceptor. Biochem Biophys Res Commun 2012; 417: 646-650.

17. Simhadri VR, Andersen JF, Calvo E, Choi SC, Coligan JE, Borrego F. Human CD300a binds to phosphatidylethanolamine and phosphatidylserine, and modulates the phagocytosis of dead cells. Blood 2012; 119: 2799-2809.

18. Choi SC, Simhadri VR, Tian L, Gil-Krzewska A, Krzewski K, Borrego $F$ et al. Cutting edge: Mouse CD300f (CMRF-35-like molecule-1) recognizes outer membraneexposed phosphatidylserine and can promote phagocytosis. J Immunol 2011; 187: 3483-3487.

19. Tian L, Choi SC, Murakami Y, Allen J, Morse lii HC, Qi CF et al. P85 $\alpha$ recruitment by the CD300f phosphatidylserine receptor mediates apoptotic cell clearance required for autoimmunity suppression. Nat Commun 2014; 5: 3146.

20. Borrego $F$. The CD300 molecules: An emerging family of regulators of the immune system. Blood 2013; 121: 1951-1960.

21. Yamanishi Y, Kitaura J, Izawa K, Matsuoka T, Oki T, Lu Y et al. Analysis of mouse LMIR5/CLM-7 as an activating receptor: Differential regulation of LMIR5/CLM-7 in mouse versus human cells. Blood 2008; 111: 688-698.

22. Yamanishi $\mathrm{Y}$, Takahashi M, Izawa $\mathrm{K}$, Isobe $\mathrm{M}$, Ito $\mathrm{S}$, Tsuchiya $\mathrm{A}$ et al. A soluble form of LMIR5/CD300b amplifies lipopolysaccharide-induced lethal inflammation in sepsis. J Immunol 2012; 189: 1773-1779.

23. Yamanishi $Y$, Kitaura J, Izawa K, Kaitani A, Komeno $Y$, Nakamura $\mathrm{M}$ et al. Tim1 is an endogenous ligand for LMIR5/CD300b: LMIR5 deficiency ameliorates mouse kidney ischemia/reperfusion injury. J Exp Med 2010; 207: 1501-1511.

24. Cannon JP, O'Driscoll M, Litman GW. Specific lipid recognition is a general feature of CD300 and trem molecules. Immunogenetics 2012; 64: 39-47.

25. Izawa K, Yamanishi Y, Maehara A, Takahashi M, Isobe M, Ito S et al. The receptor LMIR3 negatively regulates mast cell activation and allergic responses by binding to extracellular ceramide. Immunity 2012; 37: 827-839.

26. Nakahashi-Oda C, Tahara-Hanaoka S, Shoji M, Okoshi Y, Nakano-Yokomizo T, Ohkohchi $\mathrm{N}$ et al. Apoptotic cells suppress mast cell inflammatory responses via the CD300a immunoreceptor. J Exp Med 2012; 209: 1493-1503.

27. Toda S, Hanayama R, Nagata S. Two-step engulfment of apoptotic cells. Mol Cell Biol 2012; 32: 118-125.

28. Turnbull IR, Colonna M. Activating and inhibitory functions of DAP12. Nat Rev Immunol 2007; 7: 155-161.

29. Iwaszko M, Bogunia-Kubik K. Clinical significance of the HLA-E and CD94/NK $\gamma 2$ interaction. Arch Immunol Ther Exp (Warsz) 2011; 59: 353-367.

30. Linsley PS, Greene JL, Tan P, Bradshaw J, Ledbetter JA, Anasetti C et al. Coexpression and functional cooperation of CTLA-4 and CD28 on activated t lymphocytes. J Exp Med 1992; 176: 1595-1604.

31. Parham P. Killer cell immunoglobulin-like receptor diversity: Balancing signals in the natura killer cell response. Immunol Lett 2004; 92: 11-13.
32. Takai T. Paired immunoglobulin-like receptors and their MHC class I recognition. Immunology 2005; 115: 433-440.

33. Kubagawa H, Chen CC, Ho LH, Shimada TS, Gartland L, Mashburn C et al. Biochemical nature and cellular distribution of the paired immunoglobulin-like receptors, PIR- $\alpha$ and PIR- $\beta$. J Exp Med 1999; 189: 309-318.

34. Xi H, Katschke Jr KJ, Helmy KY, Wark PA, Kljavin N, Clark H et al. Negative regulation of autoimmune demyelination by the inhibitory receptor CLM-1. J Exp Med 2010; 207: 7-16.

35. Wever KE, Wagener FA, Frielink C, Boerman OC, Scheffer GJ, Allison A et al. Diannexin protects against renal ischemia reperfusion injury and targets phosphatidylserines in ischemic tissue. PLoS One 2011; 6: e24276.

36. Teoh NC, Ito Y, Field J, Bethea NW, Amr D, McCuskey MK et al. Diannexin, a novel Annexin $\mathrm{V}$ homodimer, provides prolonged protection against hepatic ischemia-reperfusion injury in mice. Gastroenterology 2007; 133: 632-646.

37. Stearns-Kurosawa DJ, Osuchowski MF, Valentine C, Kurosawa S, Remick DG. The pathogenesis of sepsis. Annu Rev Pathol 2011; 6: 19-48.

38. Rothlin CV, Lemke G. Tam receptor signaling and autoimmune disease. Curr Opin Immunol 2010; 22: 740-746.

39. Albacker LA, Yu S, Bedoret D, Lee WL, Umetsu SE, Monahan S et al. Tim4, expressed by medullary macrophages, regulates respiratory tolerance by mediating phagocytosis of antigen-specific T cells. Mucosal Immunol 2013; 6: 580-590

40. Flannagan RS, Canton J, Furuya W, Glogauer M, Grinstein S. The phosphatidylserine receptor Tim4 utilizes integrins as coreceptors to effect phagocytosis. Mol Biol Cell 2014; 25: 1511-1522

41. Baghdadi M, Yoneda A, Yamashina T, Nagao H, Komohara Y, Nagai S et al. Tim4 glycoprotein-mediated degradation of dying tumor cells by autophagy leads to reduced antigen presentation and increased immune tolerance. Immunity 2013; 39: 1070-1081.

42. Hochreiter-Hufford AE, Lee CS, Kinchen JM Sokolowski JD, Arandjelovic S, Call JA et al. Phosphatidylserine receptor BAl1 and apoptotic cells as new promoters of myoblast fusion. Nature 2013; 497: 263-267.

43. Elliott MR, Zheng S, Park D, Woodson RI, Reardon MA, Juncadella IJ et al. Unexpected requirement for ELMO1 in clearance of apoptotic germ cells in vivo. Nature 2010; 467: 333-337.

44. Park SY, Kang KB, Thapa N, Kim SY, Lee SJ, Kim IS. Requirement of adaptor protein GULP during Stabilin-2-mediated cell corpse engulfment. J Biol Chem 2008; 283 10593-10600.

45. Nishi C, Toda S, Segawa K, Nagata S. Tim4- and MerTK-mediated engulfment of apoptotic cells by mouse resident peritoneal macrophages. Mol Cell Biol 2014; 34: 1512-1520.

46. Fadok VA, Bratton DL, Konowal A, Freed PW, Westcott JY, Henson PM. Macrophages that have ingested apoptotic cells in vitro inhibit proinflammatory cytokine production through autocrine/paracrine mechanisms involving TGF- $\beta$, PGE2, and PAF. J Clin Invest 1998; 101: $890-898$.

47. Jehle AW, Gardai SJ, Li S, Linsel-Nitschke P, Morimoto K, Janssen WJ et al. ATP-binding cassette transporter a7 enhances phagocytosis of apoptotic cells and associated ERK signaling in macrophages. J Cell Biol 2006; 174: 547-556

48. Osada Y, Shiratsuchi A, Nakanishi Y. Involvement of mitogen-activated protein kinases in class b Scavenger Receptor type l-induced phagocytosis of apoptotic cells. Exp Cell Res 2006; 312: 1820-1830.

49. Hupfeld S, Holsaeter AM, Skar M, Frantzen CB, Brandl M. Liposome size analysis by dynamic/static light scattering upon size exclusion-/field flow-fractionation. J Nanosci Nanotechnol 2006; 6: 3025-3031.

50. Choi SC, Wang H, Tian L, Murakami Y, Shin DM, Borrego F et al. Mouse IgM Fc receptor, FCMR, promotes $B$ cell development and modulates antigen-driven immune responses. J Immunol 2013; 190: 987-996.

\section{Supplementary Information accompanies this paper on Cell Death and Differentiation website (http://www.nature.com/cdd)}

